Wannier-like functions and tight-binding parametrization for the manganese bands in $\mathrm{CaMnO}_{3}$

This article has been downloaded from IOPscience. Please scroll down to see the full text article.

2003 J. Phys.: Condens. Matter 151685

(http://iopscience.iop.org/0953-8984/15/10/316)

View the table of contents for this issue, or go to the journal homepage for more

Download details:

IP Address: 128.206.162.204

The article was downloaded on 09/12/2010 at 17:42

Please note that terms and conditions apply. 


\title{
Wannier-like functions and tight-binding parametrization for the manganese bands in $\mathrm{CaMnO}_{3}$
}

\author{
T Saha-Dasgupta ${ }^{1}$ and S Satpathy ${ }^{2}$ \\ ${ }^{1} \mathrm{~S}$ N Bose National Centre for Basic Sciences, JD Block, Sector 3, Salt Lake City, \\ Kolkata 700098, India \\ ${ }^{2}$ Department of Physics and Astronomy, University of Missouri, Columbia, MO 65211, USA
}

Received 25 November 2002, in final form 22 January 2003

Published 3 March 2003

Online at stacks.iop.org/JPhysCM/15/1685

\begin{abstract}
We study the electronic band structure of $\mathrm{CaMnO}_{3}$, in order to understand the origin of the dispersion of the $\operatorname{Mn}\left(e_{g}\right)$ bands, which is in contrast with the predicted dispersionless bands within the Anderson-Hasegawa doubleexchange model with infinite Hund's-rule coupling. A downfolding technique within the newly developed muffin-tin orbital-based method is used to analyse the density-functional band structure obtained in the local spin density approximation. The finite Hund's coupling parameter in realistic situations allows the same-spin bands on the two manganese sublattices to mix producing a large dispersion. The calculated Wannier functions for the $\mathrm{Mn}\left(\mathrm{e}_{\mathrm{g}}\right)$ bands also show large oxygen character at sites further away from nearest oxygen sites causing long-ranged $\mathrm{Mn}-\mathrm{Mn}$ hopping processes.
\end{abstract}

\section{Introduction}

Knowledge about perovskite manganese oxides dates back to 1950 s, with the pioneering work of Jonker and van Santen [1] establishing the close relation between the magnetism and electrical transport. The discovery of a very large negative magnetoresistance, namely the colossal magnetoresistance (CMR), has resulted in a great deal of attention being paid to these perovskite materials in recent years, particularly in the light of the exchange mechanism, and the manner in which charge, spin, and lattice degrees of freedom are coupled [2]. $\mathrm{La}_{1-x} \mathrm{D}_{x} \mathrm{MnO}_{3}$ (D being a divalent alkaline earth: $\mathrm{Ca}, \mathrm{Sr}$, or $\mathrm{Ba}$ ) systems provide a prime example of such class of compounds. The end-members ( $x=0$ and 1) are antiferromagnetic (AF) insulators. With increase in $x$, they become ferromagnetic (FM) with well-defined Curie temperatures. Around the same temperature, they also exhibit metal-like conductivity. This simultaneous occurrence of ferromagnetism and conductivity in these doped compounds was interpreted quite early on as being caused by the double-exchange mechanism [3], where the magnetic coupling between localized spins on neighbouring atoms is mediated via the conduction electrons connecting the parallel alignment of Mn moments with the hopping of carriers. Many of the studies are 
therefore based on the Anderson-Hasegawa double-exchange model [4] with infinite Hund'srule coupling, $J_{H}$, giving rise to an effective hopping integral for neighbouring spins at the angle $\theta$ of $-t \cos (\theta / 2)$ ( $t$ is the nearest-neighbour (NN) hopping integral for hopping between orbitals of same spin), implying zero kinetic energy gain in the AF Néel state and maximum kinetic energy gain in the FM state.

To investigate the scope and limitations of such commonly used infinite-Hund's-rulecoupling models, we have considered in the present paper a particular end-member of this family of compounds, namely the $\mathrm{AF} \mathrm{CaMnO}_{3}$. We have studied its electronic structure in terms of a first-principles Wannier-like description of the Mn $\mathrm{e}_{\mathrm{g}}$ bands using the newly formulated and implemented NMTO technique [5]. This compound deserves attention in its own right, being a simple case of an AF compound which can be electron doped. $\mathrm{CaMnO}_{3}$, being less prone to chemical defects than $\mathrm{LaMnO}_{3}$ [6], provides an ideal subject for detailed doping studies. Furthermore, recent conjectures about magnetic polaron formation [7-9] in these compounds with low levels of electron doping has attracted new interest. Our study provides Wannier functions and the corresponding hopping integrals of the tight-binding (TB) Mn $\mathrm{e}_{\mathrm{g}}$ bands derived from ab initio density-functional calculations. This should prove useful for realistic modelling of the manganites.

\section{Crystal structure}

The structure of $\mathrm{CaMnO}_{3}$ is relatively simple compared to those of other members of the $\mathrm{La}_{1-x} \mathrm{Ca}_{x} \mathrm{MnO}_{3}$ family. It forms in the cubic perovskite $\mathrm{ABX}_{3}$ structure, with a lattice constant of $3.73 \AA$ [10]. Each Mn atom is sixfold coordinated by oxygen atoms, thereby providing the octahedral environment at the Mn site, resulting in the splitting of the degenerate d levels into $\mathrm{t}_{2 \mathrm{~g}}$ and $\mathrm{e}_{\mathrm{g}}$ states. Considering the nominal chemical formula of $\mathrm{Ca}^{2+} \mathrm{Mn}^{4+} \mathrm{O}_{3}^{2-}$ and the fact that $\mathrm{Mn}^{4+}$ is not a Jahn-Teller-active ion, the Jahn-Teller distortion of the MnO octahedron observed in other members of the family is absent in $\mathrm{CaMnO}_{3}$. Ca ions are twelvefold coordinated by oxygen atoms lying along its $\langle 110\rangle$ directions. The $\mathrm{AF}$ insulator $\mathrm{CaMnO}_{3}$ is observed to be in the G-type AF state, so the spins at neighbouring Mn sites are antiferromagnetically coupled in all three directions. Figure 1 shows the $\mathrm{AF}$ structure of $\mathrm{CaMnO}_{3}$.

\section{LDA band structure}

The LDA calculation for $\mathrm{AF} \mathrm{CaMnO}_{3}$ has been carried out with linear muffin-tin orbital (LMTO) method within the framework of the atomic-sphere approximation (ASA) [11], with two formula units to account for the G-type magnetic unit cell. We have used the TB form of the LMTO method for the present calculation, as discussed in [12]. The orbital-projected $\mathrm{AF}$ band structure is shown in figure 2. The calculated band structure agrees with the previous calculations $[13,14]^{3}$. In figure 2 (and also in figure 3 discussed below), the $k$-points correspond to the BZ of face-centred cubic (FCC) structure appropriate for $\mathrm{AF} \mathrm{CaMnO}_{3}$, namely, $\Gamma=(0,0,0), \mathrm{X}=(2 \pi / a)\left(\frac{1}{2 \sqrt{(2)}}, \frac{1}{2 \sqrt{(2)}}, 0\right), \mathrm{W}=(2 \pi / a)\left(\frac{3}{4 \sqrt{(2)}}, \frac{1}{4 \sqrt{(2)}}, 0\right)$, and $\mathrm{L}=(2 \pi / a)\left(\frac{1}{2 \sqrt{(2)}}, 0,1 / 4\right)$ with the $x$ - and $y$-coordinate axes chosen along the diagonal MnMn direction and the $z$-axes chosen along the perpendicular Mn-O-Mn direction (as shown in figure 1); $a$ is the cubic lattice constant equal to $3.73 \AA$. Shown in the figure are projections

\footnotetext{
${ }^{3}$ In this paper, the $\mathrm{W}$ point for $\mathrm{AF} \mathrm{CaMnO}_{3}$ was different from the conventional $\mathrm{W}$ point used in the present paper. Also, the $\mathrm{X}$ point was approached from $\Gamma$ using a direction different from the $\Delta$ one. The $k$-points used were $\mathrm{X}=(2 \pi / a)\left(0, \frac{1}{\sqrt{(2)}}, 0\right), \mathrm{W}=(2 \pi / a)\left(\frac{1}{4 \sqrt{(2)}}, \frac{5}{4 \sqrt{(2)}},-1 / 4\right)$, and $\mathrm{L}=(2 \pi / a)\left(\frac{1}{2 \sqrt{(2)}}, \frac{1}{\sqrt{(2)}}, 0\right)$
} 


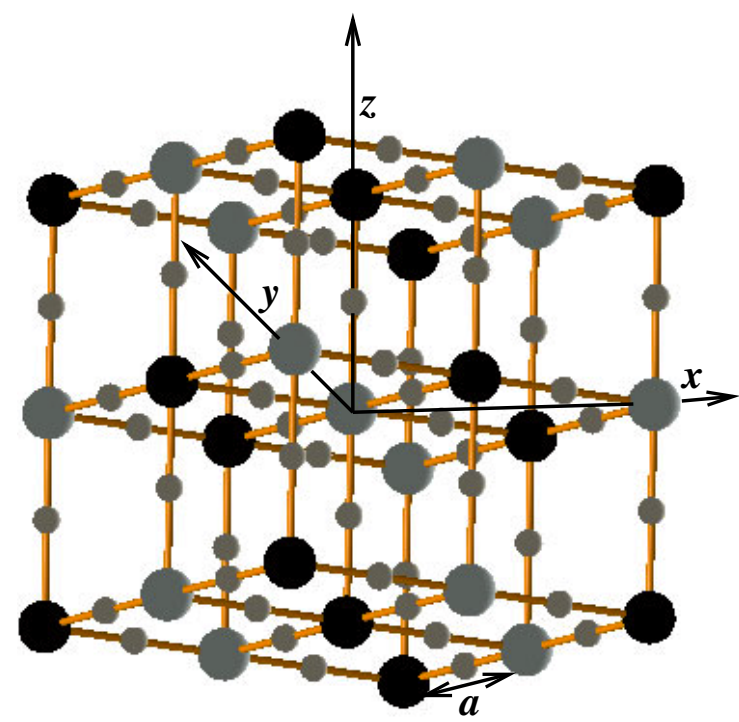

Figure 1. The G-type AF structure of $\mathrm{CaMnO}_{3}$. Large black and grey balls denote the Mn atoms belonging to two different AF sublattices, while the small balls denote the oxygen sites. Ca sites have not been shown, for clarity.

(This figure is in colour only in the electronic version)
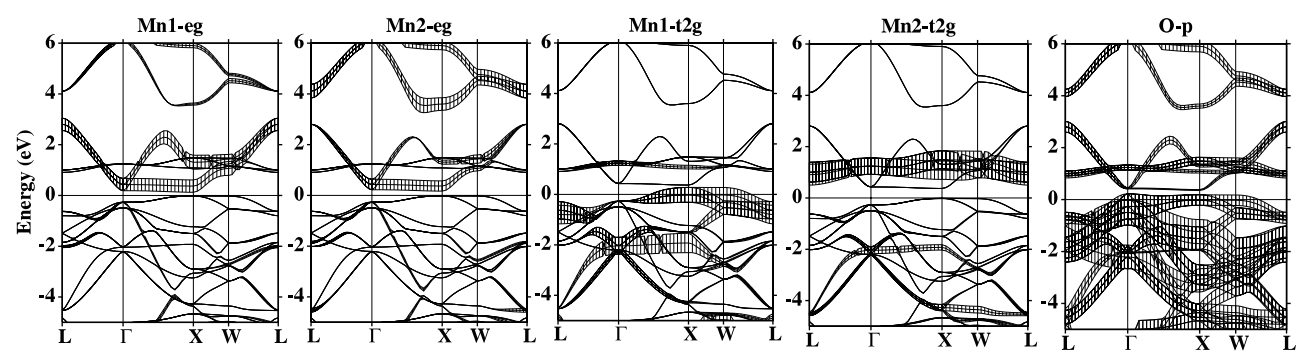

Figure 2. The orbital-decomposed band structure of $\mathrm{AF} \mathrm{CaMnO}_{3}$.

to $\mathrm{e}_{\mathrm{g}}{ }^{-}, \mathrm{t}_{2 \mathrm{~g}}$-derived states at the two Mn sites (denoted as Mn1 and Mn2) belonging to the two different sublattices of the AF structure and that of the oxygen $\mathrm{p}$ states. The width of the bands in each panel is proportional to the weight of the indicated orbital in the wavefunction. By AF construction, the state in the up-spin channel at the Mn1 site is equivalent to that in the down-spin channel at the Mn2 site and vice versa. While $\mathrm{e}_{\mathrm{g}}$-derived states at the Mn1 site span the energy range of about $0.2-2.5 \mathrm{eV}$, the $\mathrm{e}_{\mathrm{g}}$-derived states at the Mn2 site span the energy range of about 3.5-6 eV. The $\mathrm{t}_{2 \mathrm{~g}}$-derived states at the Mn2 site appear at an energy of about $1.5 \mathrm{eV}$, in the middle of the range of the $\mathrm{e}_{\mathrm{g}}$-derived Mn1 states. The significant feature to notice is the appreciable dispersion of the $\mathrm{e}_{\mathrm{g}}$-derived bands and the non-negligible oxygen contributions in these bands. We note that the Anderson-Hasegawa model of infinite Hund'srule coupling with effective hopping of $-t \cos (\theta / 2)$ would give rise to dispersionless $\mathrm{Mn} \mathrm{e}_{\mathrm{g}}$ bands, since the term $-t \cos (\theta / 2)$ would prohibit any processes of hopping between spins that are antiferromagnetically aligned. 

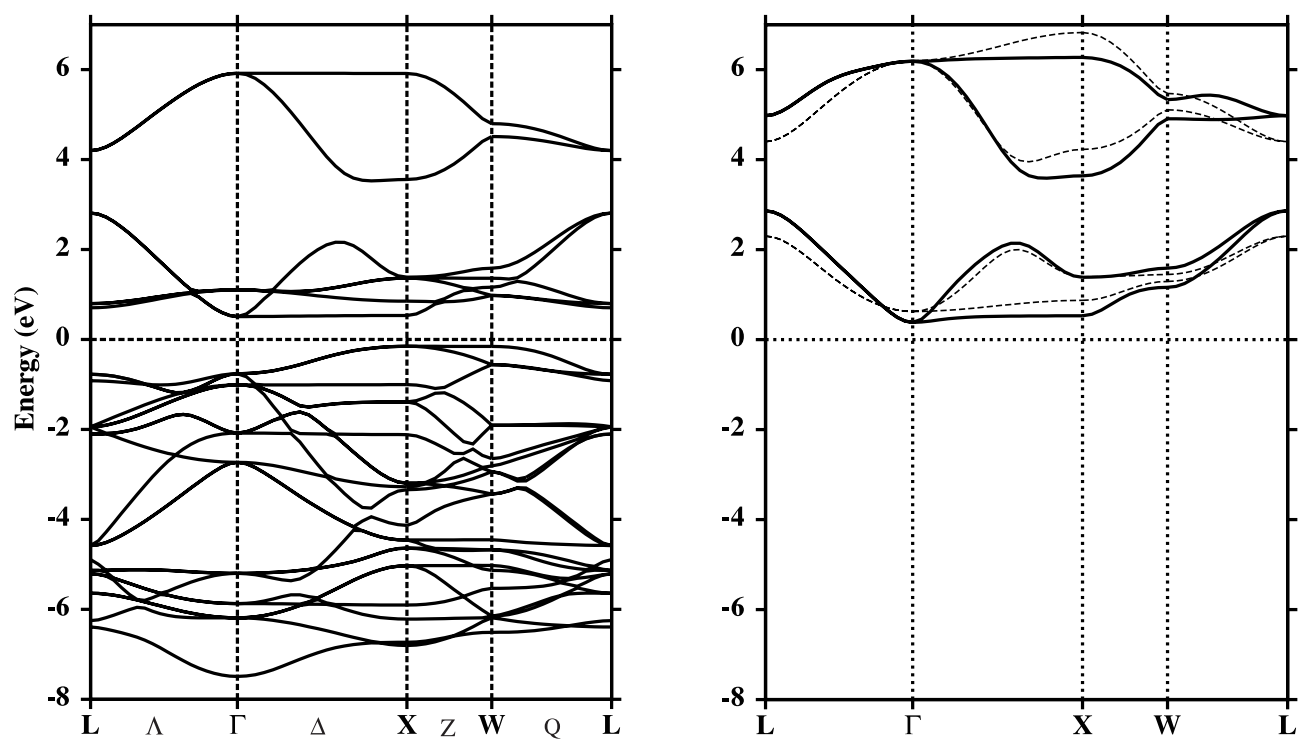

Figure 3. The full LDA band structure (left panel) and the downfolded Mn $\mathrm{e}_{\mathrm{g}}$-only bands (right panel) for $\mathrm{AF} \mathrm{CaMnO}_{3}$. The right panel also shows, as dashed lines, the best-fit TB bands for first- and second-NN hoppings, with the parameters given in table 1 .

\section{Downfolding: Wannier-like orbitals and the tight-binding Hamiltonian}

In recent years, the downfolding technique within the framework of an improved LMTO method, namely the $N$ th-order MTO (NMTO) method [5], has been developed, a method that goes beyond the standard LMTO method in describing an energetically accurate basis set with a consistent description throughout the space of MT spheres and the interstitials. The new method provides a useful way of deriving the few-band Hamiltonians starting from complicated LDA band structure. It has been successfully applied to systems such as low-dimensional magnetic compounds [15, 16], high- $T_{c}$ compounds [17], and double perovskites [18]. This method, which relies on integrating out the other degrees of freedom, keeping only the relevant degrees of freedom or degrees of freedom of interest, naturally takes into account the renormalization effect due to the integrated orbitals in the effective orbitals defining the few-band Hamiltonian. Fourier transformation of this few-orbital downfolded Hamiltonian provides the hopping matrix elements of the corresponding TB Hamiltonian defined in the effective orbital basis, while the effective orbitals themselves provide the Wannier-like functions of the corresponding bands. It is to be noted here that this method provides a first-principles way of deriving the single-particle model Hamiltonian and directly generating Wannier-like functions without any fitting procedure. This way of generating Wannier-like functions may be contrasted with that starting from the Bloch-function outputs of some standard band-structure method and then constructing them demanding that the maximum localization property be exhibited [19].

In the present study we have applied this technique to derive an effective Hamiltonian with only the $\operatorname{Mn}\left(e_{g}\right)$ orbitals, which will help with the understanding of the origin of the appreciable dispersional width of the $\mathrm{Mn} \mathrm{e}_{\mathrm{g}}$ bands as provided by LDA calculations bringing in the important interaction pathways. In the right-hand panel of figure 3 we show (as solid curves) the bands obtained by downfolding all the other channels except the Mn $\mathrm{e}_{\mathrm{g}}$ channels. For comparison, in the left-hand panel of figure 3 the full LDA band structure is shown. We 

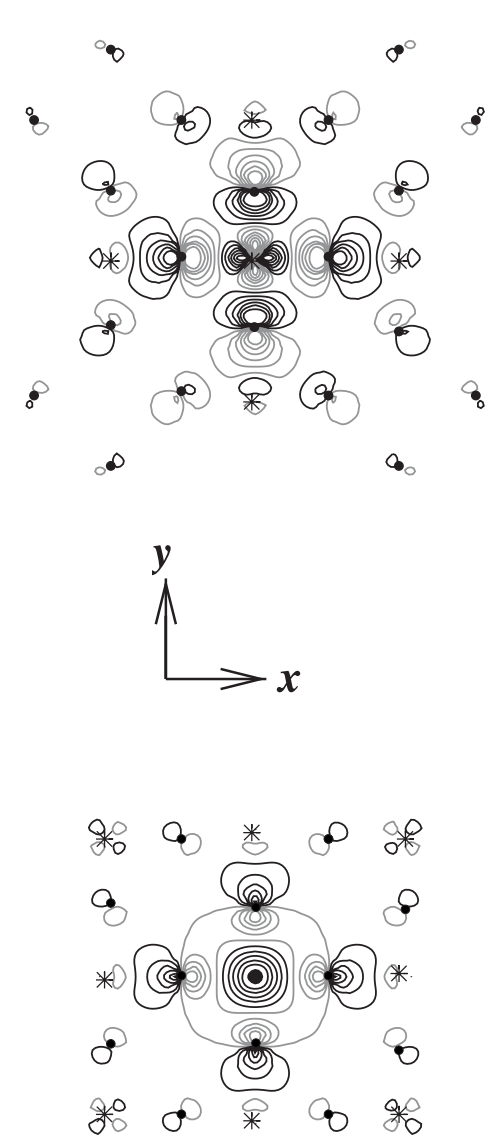

* Mn

- $\mathbf{O}$
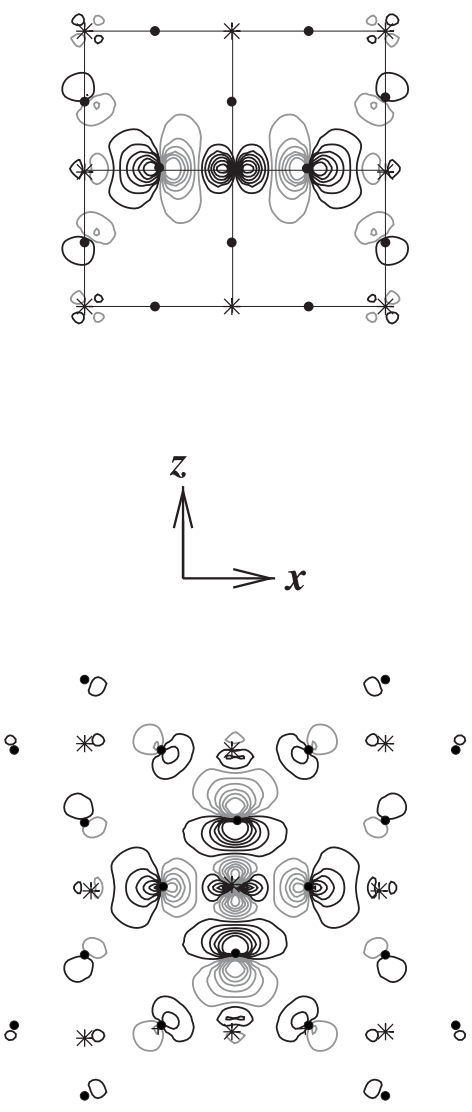

Figure 4. Downfolded $\operatorname{Mn} x^{2}-y^{2}$ (upper panel) and $3 z^{2}-1$ (lower panel) orbitals plotted in $x y$ and $x z$-planes. The darker and lighter curves represent positive and negative lobes of the orbitals respectively. The underlying $\mathrm{Mn}-\mathrm{O}$ lattice is also shown in the upper right panel.

note the efficiency of the downfolding method in picking out the correct $\mathrm{Mg} \mathrm{e}_{\mathrm{g}}$-like bands from the overlapping $\mathrm{Mn} 1 \mathrm{e}_{\mathrm{g}}$ and $\mathrm{Mn} 2 \mathrm{t}_{2 \mathrm{~g}}$ band complex. The effective Mn $\mathrm{e}_{\mathrm{g}}$ MTOs which form the Wannier-like functions corresponding to downfolded $\mathrm{Mn} \mathrm{e}_{\mathrm{g}}$ bands are shown in figure 4 . The $\mathrm{e}_{\mathrm{g}}$ orbitals with $x^{2}-y^{2}$ and $3 z^{2}-1$ symmetry at the Mn1 site have been plotted in the $x y$ - and $x z$-planes. The tails of these effective orbitals are shaped according to the renormalization effect due to the contribution of the orbitals being downfolded. We note the significant presence of oxygen character in the tails of the effective orbitals further away from NN oxygen sites, giving rise to the long-range nature of these orbitals. We also note the presence of $\mathrm{t}_{2 \mathrm{~g}}$ character at the $\mathrm{Mn}$ sites due to the overlap of the $\mathrm{t}_{2 \mathrm{~g}}$ - and $\mathrm{e}_{\mathrm{g}}$-derived states at the two Mn sublattices.

The effective TB Hamiltonian is of dimension $4 \times 4$, defined on the basis of two effective $\mathrm{e}_{\mathrm{g}}$ orbitals for each of the two Mn sites belonging to two magnetic sublattices. Fourier transformation of the downfolded Hamiltonian $(H(k) \rightarrow H(R))$ gives a TB electronic Hamiltonian in real space consisting of hopping over up to five NNs. For many studies, it is desirable to have a shorter-range Hamiltonian. In order to arrive at a short-ranged Hamiltonian, restricted to second $\mathrm{NN}$, and still keeping the advantage of the downfolding method in properly 
Table 1. The Slater-Koster parameters for the best fit corresponding to the $2 \mathrm{NN}$ TB Hamiltonian for $\mathrm{Mn} \mathrm{e}_{\mathrm{g}}$ bands of $\mathrm{AF} \mathrm{CaMnO}_{3}$. For the $\mathrm{NN}$ interaction in a simple cubic lattice, there is no dd $\pi$ interaction with $\mathrm{e}_{\mathrm{g}}$ orbitals and $V_{\mathrm{dd} \pi}$ does not appear [16].

\begin{tabular}{lllll}
\hline On-site: & Distance $=0.00 a$ & $\epsilon_{\mathrm{e}_{g}}^{\mathrm{Mn} 1}=2.30 \mathrm{eV}$ & $\epsilon_{\mathrm{e}_{g}}^{\mathrm{Mn} 2}=4.40 \mathrm{eV}$ & \\
$1 \mathrm{NN}:$ & Distance $=1.00 a$ & & & \\
& Mn1-Mn2 & $\mathrm{dd} \sigma=-0.90 \mathrm{eV}$ & & $\mathrm{dd} \delta=0.07$ \\
$2 \mathrm{NN}:$ & Distance $=1.41 a$ & & & \\
& Mn1-Mn1 & $\mathrm{dd} \sigma=-0.25 \mathrm{eV}$ & $\mathrm{dd} \pi=0.04 \mathrm{eV}$ & $\mathrm{dd} \delta=0.00 \mathrm{eV}$ \\
& $\mathrm{Mn} 2-\mathrm{Mn} 2$ & $\mathrm{dd} \sigma=-0.20 \mathrm{eV}$ & $\mathrm{dd} \pi=0.10 \mathrm{eV}$ & $\mathrm{dd} \delta=-0.01 \mathrm{eV}$ \\
\hline
\end{tabular}

Table 2. The Slater-Koster parameters for the best fit corresponding to $2 \mathrm{NN}$ TB Hamiltonian for $\mathrm{Mn} \mathrm{e}_{\mathrm{g}}$ bands of $\mathrm{FM} \mathrm{CaMnO}_{3}$

\begin{tabular}{|c|c|c|c|c|}
\hline On-site: & Distance $=0.00 a$ & $\epsilon_{\mathrm{e}_{g}}^{\mathrm{Mn}}=2.00 \mathrm{eV}$ & & \\
\hline \multirow[t]{2}{*}{$1 \mathrm{NN}:$} & Distance $=1.00 a$ & & & \\
\hline & & $\mathrm{dd} \sigma=-0.75 \mathrm{eV}$ & & $\operatorname{dd} \delta=0.08$ \\
\hline \multirow[t]{2}{*}{$2 \mathrm{NN}:$} & Distance $=1.41 a$ & & & \\
\hline & & $\mathrm{dd} \sigma=-0.27 \mathrm{eV}$ & $\mathrm{dd} \pi=0.04 \mathrm{eV}$ & $\mathrm{dd} \delta=0.03 \mathrm{eV}$ \\
\hline
\end{tabular}

taking into account the renormalization effect, we have used a combined method where we have used the optimization procedure to reproduce the downfolded bands starting from the hopping matrix elements provided by the Fourier transform of the downfolded Hamiltonian as the input. The second-NN Hamiltonian that best fits the downfolded bands expressed in terms of the Slater-Koster integrals $\mathrm{dd} \sigma, \mathrm{dd} \pi, \mathrm{dd} \delta$ (by turning the $z$-axis along the line connecting two interacting sites) are shown in table 1. The corresponding TB bands are shown by dashed curves in the right-hand panel of figure 3.

The on-site energy difference between the $\mathrm{e}_{\mathrm{g}}$ states at two Mn sites, Mn1 and Mn2, belonging to two magnetic sublattices, which is caused by the exchange splitting between the spin channels at a given Mn site (by AFM construction), turns out to be finite, and moderate in value $(\approx 2.1 \mathrm{eV})$, in contrast with the assumption of infinite Hund's-rule coupling. This estimate of the on-site energy difference between two effective $\mathrm{Mg}$ e $_{\mathrm{g}}$ levels at two magnetic sublattices may be considered as a lower bound for the Hund's-rule coupling in this material. In the absence of the strong renormalization effect from oxygen p levels, this could have been considered as an estimate of the Hund's-rule coupling. However, the hybridization from the oxygen $\mathrm{p}$ levels pushes the $\mathrm{Mn} \mathrm{e}_{\mathrm{g}}$ levels in the effective $\mathrm{Mn} \mathrm{e}_{\mathrm{g}}$ model, giving rise to a lower value of this energy difference compared to that obtained from the constrained LDA kind of approach [14].

At the end, we also carried out similar analysis with $\mathrm{CaMnO}_{3}$ in the hypothetical FM phase, since this is of relevance for the study of electron-doped systems, where local FM order may be induced by the doped electrons (magnetic polarons). The FM phase has higher energy compared to the ground-state AF phase. Figure 5 shows the LDA bands (left-hand panel) and the downfolded $\mathrm{e}_{\mathrm{g}}$-only bands (as solid curves, right-hand panel) for the majority spin channel. The $k$-points are: $\Gamma=(0,0,0), \mathrm{X}=(2 \pi / a)(0,1 / 2,0)$, and $\mathrm{M}=(2 \pi / a)(1 / 2,1 / 2,0)$. The right-hand panel of the figure also shows the TB bands with nearest- and next-nearest-neighbour hoppings, as dashed curves. The TB parameters obtained are given in table 2.

The NN hopping obtained by Chen and Allen [8] is consistent with ours, although, as seen from table 2, we also find a significant next-nearest-neighbour hopping. 

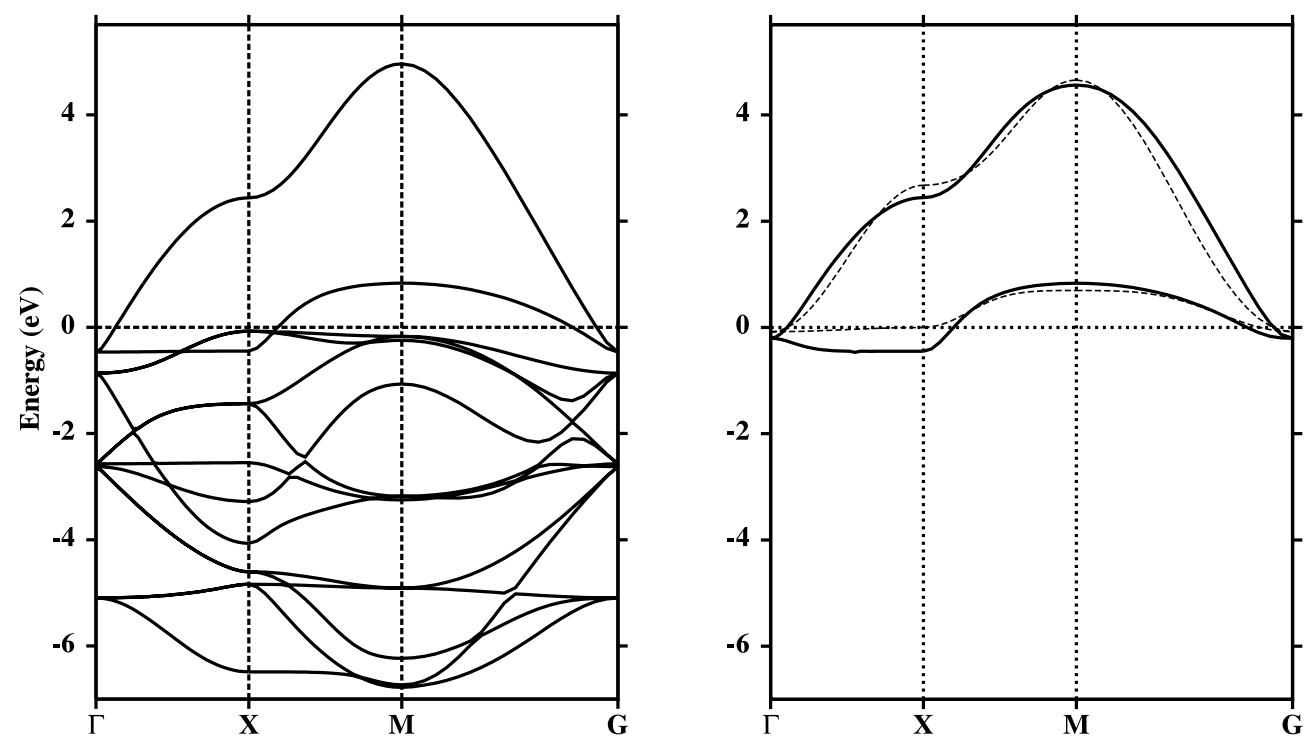

Figure 5. The full LDA band structure (left panel) and the downfolded Mn $\mathrm{e}_{\mathrm{g}}$-only bands (right panel) in the majority spin channel for $\mathrm{FM} \mathrm{CaMnO}_{3}$. The right-hand panel also shows, as dashed curves, the best-fit TB bands with first- and second-NN hoppings with the parameters given in table 2 .

\section{Conclusions}

In summary, by using the downfolding technique within the new formulation of the NMTO method, we have analysed the nature of the $\mathrm{Mn} \mathrm{e}_{\mathrm{g}}$-derived bands in $\mathrm{CaMnO}_{3}$ in terms of Wannier-like functions and obtained the parameters for the TB Hamiltonian. The effective $\mathrm{Mn} \mathrm{e}_{\mathrm{g}}$ orbitals for $\mathrm{CaMnO}_{3}$ obtained by integrating out the other degrees of freedom show significant long-ranged oxygen characters, and $\mathrm{Mn} \mathrm{t}_{2 \mathrm{~g}}$ characters at other sublattices, caused by the moderate value of the Hund's-rule coupling parameter $J_{H}$ allowing mixing between the two sublattices. This is turn gives rise to the appreciable width of the $\mathrm{Mn} \mathrm{e}_{\mathrm{g}}$ bands in $\mathrm{CaMnO}_{3}$. We have also carried out a downfolding calculation to derive the $\mathrm{Mn} \mathrm{e}_{\mathrm{g}}$ bands in the hypothetical FM phase of $\mathrm{CaMnO}_{3}$ and obtained the corresponding parameters for the TB Hamiltonian.

\section{Acknowledgments}

This work was jointly supported by the Department of Science and Technology, India, and the National Science Foundation, USA, under the project No DST-NSF/RPO-57/2001.

\section{References}

[1] Jonker G H and van Santen J H 1950 Physica 16377

[2] Rao C N R, Cheetham A K and Mahesh R 1996 Chem. Mater. 82421

[3] Zener C 1951 Phys. Rev. 82403

[4] Anderson P W and Hasegawa H 1955 Phys. Rev. 100675

[5] Andersen O K and Saha-Dasgupta T 2000 Phys. Rev. B 62 R16219 and references therein

[6] van Roosmalen A M and Cordfunke E H P 1994 J. Solid State Chem. 110109

[7] Neumeier J J and Cohn J L 2000 Phys. Rev. B 6114319 
[8] Chen Y and Allen P B 2001 Phys. Rev. B 64064401

[9] Meskine $\mathrm{H}$ et al, unpublished

[10] Elemans J B A A, van Laar B, van der Veen K R and Loopstra B O 1971 J. Solid State Chem. 3238

[11] Andersen O K 1975 Phys. Rev. B 123060

[12] Andersen O K and Jepsen O 1984 Phys. Rev. Lett. 532571

[13] Pickett W and Singh D 1996 Phys. Rev. B 531146

[14] Satpathy S, Popović Z and Vukajlović F 1996 Phys. Rev. Lett. 76960

[15] Müller T, Anisimov V, Rice T M, Dasgupta I and Saha-Dasgupta T 1998 Phys. Rev. B 57 R12655

[16] Valentí R, Saha-Dasgupta T, Alvarez J V, Pozgajcic K and Gros C 2001 Phys. Rev. Lett. 865381

[17] Pavarini E, Dasgupta I, Saha-Dasgupta T, Jepsen O and Andersen O K 2001 Phys. Rev. Lett. 87047003

[18] Sarma D D, Mahadevan P, Saha-Dasgupta T, Ray S and Kumar A 2000 Phys. Rev. Lett. 852549

[19] Marzari N and Vanderbilt D 1997 Phys. Rev. B 5612847

[20] Walter A Harrison 1980 Electronic Structure and the Properties of Solids (San Francisco, CA: Freeman) p 481 